\title{
Electromagnetic Response of Horizontal Magnetic Dipole over Inhomogeneous Earth Model with Linear Conductivity Variation
}

\author{
Nagendra P. SINGH and T. LAL \\ Department of Geophysics, Banaras Hindu University, Varanasi-221005, India
}

(Received July 21, 1995; Revised February 9, 1996; Accepted June 29, 1996)

\begin{abstract}
The problem of calculating the electromagnetic (em) field response of a horizontal magnetic dipole placed over a multi layer earth model with one of the layers having linear variation of conductivity with depth is formulated. Analytical solutions are obtained for the three layer earth model having linear variation of conductivity in the intermediate transition layer, for the case of quasi-static approximation. Computations are performed for the absolute-amplitude ratio, i.e. the ratio of the absolute amplitude of the field component over a multi-layer model and the corresponding component over a homogeneous half space possessing the conductivity of the top layer. The effects of transition layer thickness and conductivity contrast between the top and bottom layers are investigated by computing these ratios as a function of numerical distance. The results depict characteristic dependence of the em response on conductivity inhomogeneity.
\end{abstract}

\section{Introduction}

The general trend in electromagnetic investigations is to consider the earth models consisting of a set of homogeneous layers. However, to represent the actual structural features inside the earth more closely, it is necessary to take into account the continuous variation of electrical conductivity of the medium. The presence of such inhomogeneous layers, which act as transition zone, in which conductivity changes continuously from one set of values to another has been suggested on a local as well as on a global scale. Resistivity logs (Mallick and Roy, 1968) invariably show geological sections consisting of layers in which resistivity/conductivity changes continuously providing a transition between the overlying and underlying layers. On global scale, the existence of such transition layers can be visualised by the general profile of resistivity values through earth's crust (Keller, 1971). Therefore, in general it is noticed that the discontinuities are frequent in real earth situations. These gradual variations in conductivity may be approximated by the simplest mathematical law of linear variation with depth. Thus, it seems pertinent to take into account the study of em response over inhomogeneous earth models having linear variation of conductivity with depth. Moreover, this analysis would be of much importance and very well suited to be formulated as an inversion problem in which the response from a model with linear variation in conductivity is attempted using a stack of homogeneous layers.

The study of em response over inhomogeneous earth models have attracted the attention of many scientists. Mallick and Roy (1971) have presented the analytical solution for the em field components over transition layer model possessing linear variation of conductivity with depth for the vertical magnetic dipole source, while Abramovici and Chlamtac (1978) and Chlamtac and Abramovici (1981) have presented the em response over transition layer models having linear variation of conductivity with depth for the vertical magnetic and horizontal electric dipole sources respectively.

In the present paper, the problem of calculating the em field response of a horizontal magnetic dipole placed over the surface of a multi-layer earth model with one of the layers having conductivity varying linearly with depth is studied. Analytical expressions and computational results for the various electric and magnetic field components are presented for the three layer earth models with intermediate layer possessing linear variation of conductivity with depth. 


\section{Formulation and Solution of the Problem}

One-dimensional multi layer earth model under investigation is shown in Fig. 1. The earth model comprises of a sequence of $N$-layers, of which $(N-1)$ layers are homogeneous and the $j$-th one is inhomogeneous. The cartesian coordinate system $(x, y, z)$ with its $z$ axis directed vertically upward is used. The cylindrical coordinate system $(\rho, \phi, z)$ has also been used at many places. A relative diagram of these two coordinate systems is shown in the inset of Fig. 1. A horizontal magnetic dipole, a small circular loop carrying low frequency alternating current with its plane normal to the $x$ axis, is placed at a height $h$ just vertically above the origin.

Let $\sigma_{j}, \mu_{j}, h_{j}(j=1,2, \ldots, N)$ be the conductivity, permeability and depth to the lower boundary of each layer, respectively. The conductivity and permeability of the free space are taken to be $\sigma_{0}$ and $\mu_{0}$ respectively. Further, as the magnetic permeability value for the rock formations in general equals the free space value, it is assumed that for all the layers $\mu_{j}(j=1,2, \ldots, N)=\mu_{0}$. The conductivity $\sigma_{j}$ in the $j$-th

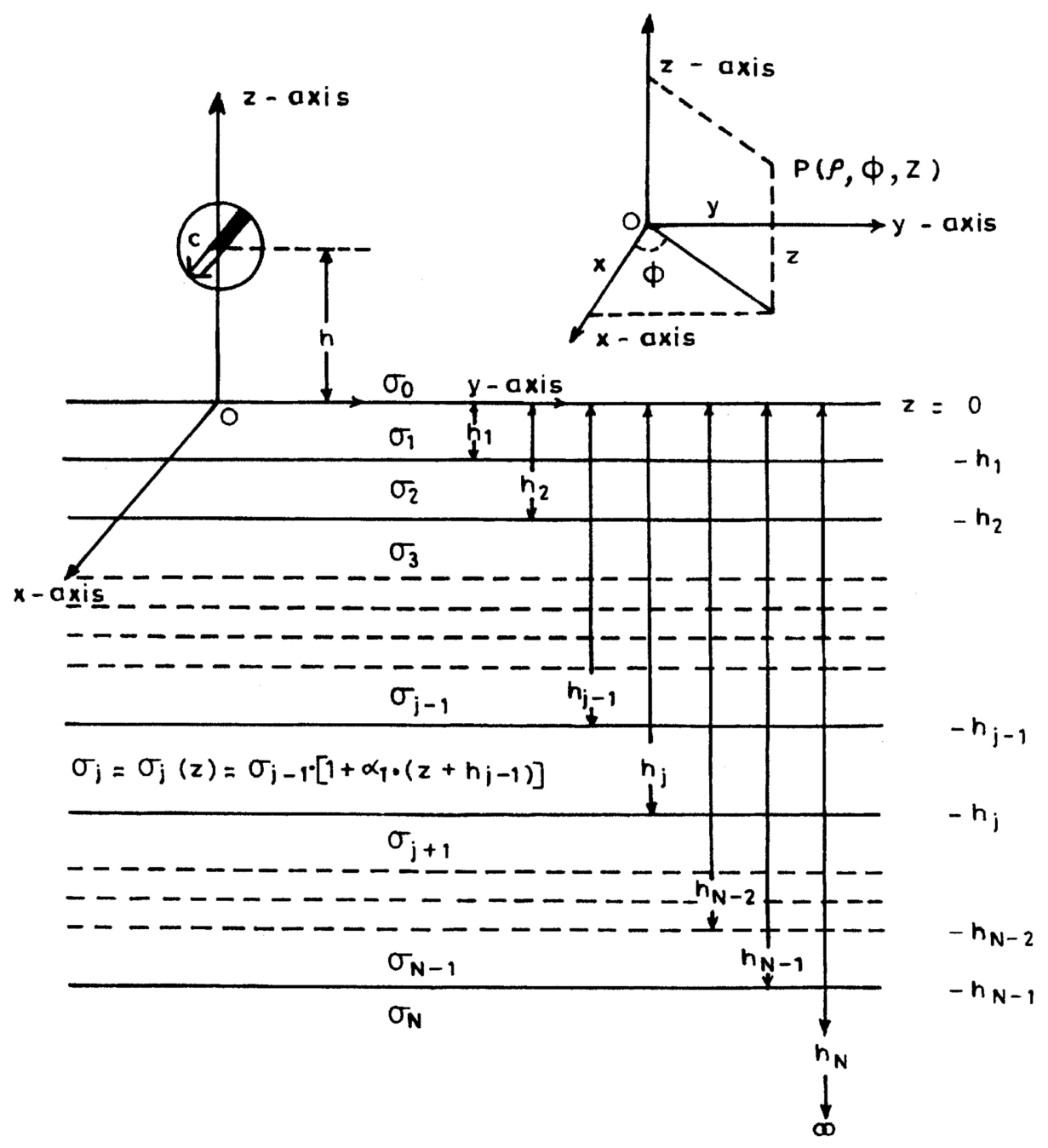

Fig. 1. Geometry of the earth model under consideration. 
inhomogeneous layer which acts as a transition zone is assumed to vary in accordance with the linear variation with depth defined by the relation:

$$
\sigma_{j}(z)=\sigma_{j-1}\left[1+\alpha_{1}\left(z+h_{j-1}\right)\right]
$$

Here the conductivity of the $(j-1)$-th layer gradually merges with that of the $(j+1)$-th layer through the $j$-th transition layer so that $\sigma_{j}(z)=\sigma_{j-1}$ at $z=-h_{j-1}$ and $\sigma_{j}(z)=\sigma_{j+1}$ at $z=-h_{j}$; and $\alpha_{1}$ is the constant dependent upon the model parameters.

The radiation constant $\gamma$ is, in general, defined as $\gamma=\left(i \omega \mu \sigma+\varepsilon \mu \omega^{2}\right)^{1 / 2}$. A harmonic time factor $e^{i \omega t}$ is implied. Further, for the low frequencies, on neglecting the displacement current, radiation constant assumes the value $\gamma=(i \omega \mu \sigma)^{1 / 2}$. The radiation constants $\gamma_{0}$ in the air and $\gamma_{1}, \gamma_{2}, \gamma_{j-1}, \gamma_{j+1}, \ldots, \gamma_{N}$ in the homogeneous layers assume constant values while $\gamma_{j}(z)$, in the transition layer varies linearly with depth in correspondence with the conductivity variation. This can be expressed as;

$$
\gamma_{j}^{2}(z)=\gamma_{j-1}^{2}\left[1+\alpha_{1}\left(z+h_{j-1}\right)\right]= \begin{cases}\gamma_{j-1}^{2} ; & \text { at } z=-h_{j-1} \\ \gamma_{j+1}^{2} ; & \text { at } z=-h_{j}\end{cases}
$$

where

$$
\alpha_{1}=\frac{1}{\left(h_{j-1}-h_{j}\right)}\left[\left(\frac{\gamma_{j+1}}{\gamma_{j-1}}\right)^{2}-1\right] \text { and } \gamma_{j}^{2}=i \omega \mu_{j} \sigma_{j}
$$

Let $d A$ be the area of the loop representing horizontal magnetic dipole and $I$ be the low frequency alternating current circulating the loop. The moment $M$ of the dipole is given by

$$
M=\frac{I d A}{4 \pi} .
$$

The primary Hertz vector potential $\left(\Pi_{p}\right)$, which has only $x$ component in the region $(z>0)$ can be written as

$$
\pi_{p}=M e^{-\gamma_{0} r} / r
$$

where

$$
\gamma_{0}=\sqrt{i \omega \mu_{0} \sigma_{0}} \text { and } r=\sqrt{\rho^{2}+|z-h|^{2}}
$$

Using Sommerfeld (1947) relations, one can write

$$
\pi_{p}=M \frac{e^{-\gamma_{0} r}}{r}=M \int_{0}^{\infty} \frac{\lambda}{n_{0}} e^{-n_{0}|z-h|} \cdot J_{0}(\lambda \rho) d \lambda
$$

where $\lambda$ is the wavenumber and $n_{0}=\sqrt{\lambda^{2}+\gamma_{0}^{2}}$. While the primary potential has only $x$ component, two 
components need to be employed for the secondary potentials. In general, the Hertz magnetic vector potential may be written as

$$
\begin{aligned}
& \Pi=i \pi_{x}+k \pi_{z}, \\
& \pi_{y}=0
\end{aligned}
$$

where $i$ and $\boldsymbol{k}$ are unit vectors in $x$ and $z$ directions respectively. The harmonic electric and magnetic fields $\boldsymbol{E}$ and $\boldsymbol{H}$ are related to the Hertz vector $\Pi$ by the relations

$$
\begin{aligned}
& \boldsymbol{E}=-i \omega \mu \cdot \operatorname{Curl\Pi ,} \\
& \boldsymbol{H}=-i \omega \mu \sigma \cdot \Pi+\operatorname{grad}(\operatorname{div} \Pi) .
\end{aligned}
$$

The components of the fields can be written directly as

$$
\begin{array}{ll}
E_{x}=i \omega \mu_{0} \cdot \frac{\partial \pi_{z}}{\partial y} ; & H_{x}=-i \omega \mu(\sigma+i \omega \varepsilon) \pi_{x}+\frac{\partial}{\partial x}\left(\frac{\partial \pi_{x}}{\partial x}+\frac{\partial \pi_{z}}{\partial z}\right) \\
E_{y}=i \omega \mu_{0}\left(\frac{\partial \pi_{x}}{\partial z}-\frac{\partial \pi_{z}}{\partial x}\right) ; & H_{y}=\frac{\partial}{\partial y}\left(\frac{\partial \pi_{x}}{\partial x}+\frac{\partial \pi_{z}}{\partial z}\right), \\
E_{z}=-i \omega \mu_{0} \cdot \frac{\partial \pi_{x}}{\partial y} ; & H_{z}=-i \omega \mu(\sigma+i \omega \varepsilon) \pi_{z}+\frac{\partial}{\partial z}\left(\frac{\partial \pi_{x}}{\partial x}+\frac{\partial \pi_{z}}{\partial z}\right) .
\end{array}
$$

The Hertz vector potential $\pi_{x}$ and $\pi_{z}$ are the solution of wave equations

$$
\nabla^{2} \pi_{x j}=\gamma_{j}^{2} \pi_{x j}
$$

and

$$
\nabla^{2} \pi_{z j}=\gamma_{j}^{2} \pi_{z j}
$$

The solution of the wave equations (10) and (11) for the air $\left(\pi_{0}\right)$, homogeneous layers $\left(\pi_{j}\right)$ and lower half space $\left(\pi_{N}\right)$ of the model with constant radiation constants can be written directly as (Ward, 1967):

$$
\begin{aligned}
& \pi_{x 0}=M \int_{0}^{\infty} \frac{\lambda}{n_{0}} e^{-n_{0}|z-h|} \cdot J_{0}(\lambda \rho) d \lambda+\int_{0}^{\infty} A(\lambda) e^{-n_{0} z} \cdot J_{0}(\lambda \rho) d \lambda, \\
& \pi_{x j}=\int_{0}^{\infty}\left\{B_{j 1}(\lambda) e^{n_{j} z}+B_{j 2}(\lambda) e^{-n_{j} z}\right\} J_{0}(\lambda \rho) d \lambda \text { for } j=1,2, \ldots, j-1, j+1, \ldots, N-1, \\
& \pi_{x N}=\int_{0}^{\infty} D(\lambda) e^{n_{N} z} \cdot J_{0}(\lambda \rho) d \lambda,
\end{aligned}
$$




$$
\begin{aligned}
& \pi_{z 0}=\frac{\partial}{\partial x} \int_{0}^{\infty} Z_{0} \cdot J_{0}(\lambda \rho) d \lambda=-\cos \phi \int_{0}^{\infty} Z_{0} \lambda \cdot J_{1}(\lambda \rho) d \lambda \\
& \pi_{z j}=\frac{\partial}{\partial x} \int_{0}^{\infty} Z_{j} \cdot J_{0}(\lambda \rho) d \lambda=-\cos \phi \int_{0}^{\infty} Z_{j} \lambda \cdot J_{1}(\lambda \rho) d \lambda \text { for } j=1,2, \ldots, j-1, j+1, \ldots, N-1, \\
& \pi_{z N}=\frac{\partial}{\partial x} \int_{0}^{\infty} Z_{N} \cdot J_{0}(\lambda \rho) d \lambda=-\cos \phi \int_{0}^{\infty} Z_{N} \lambda \cdot J_{1}(\lambda \rho) d \lambda
\end{aligned}
$$

where

$$
\begin{aligned}
& Z_{0}=P_{0}(\lambda) e^{-n_{0} z} \\
& Z_{j}=P_{j 1}(\lambda) e^{n_{j} z}+P_{j 2}(\lambda) e^{-n_{j} z} \text { for } j=1,2, \ldots, j-1, j+1, \ldots, N-1, \\
& Z_{N}=P_{N}(\lambda) e^{n_{N} z}
\end{aligned}
$$

with $\gamma_{j}=\sqrt{i \omega \mu_{j} \sigma_{j}}$ and $n_{j}=\sqrt{\lambda^{2}+\gamma_{j}^{2}}$, for all $j$. In the following, the solution of wave equations (10) and (11) for the transition layer where the conductivity varies with depth have been obtained which gives the Hertz vector potential $\pi_{x}$ and $\pi_{z}$ in the transition layer as (Mallick and Roy, 1971):

$$
\pi_{x}(z)=\int_{0}^{\infty} t^{1 / 2}\left[C_{1}(\lambda) \cdot J_{1 / 3}\left(\frac{2}{3} t^{3 / 2}\right)+C_{2}(\lambda) \cdot J_{-1 / 3}\left(\frac{2}{3} t^{3 / 2}\right)\right] J_{0}(\lambda \rho) d \lambda
$$

and

$$
\pi_{z}(z)=\frac{\partial}{\partial x} \int_{0}^{\infty} t^{1 / 2}\left[Q_{1}(\lambda) \cdot J_{1 / 3}\left(\frac{2}{3} t^{3 / 2}\right)+Q_{2}(\lambda) \cdot J_{-1 / 3}\left(\frac{2}{3} t^{3 / 2}\right)\right] J_{0}(\lambda \rho) d \lambda
$$

where

$$
t=\left(\frac{\gamma_{j-1}}{\alpha_{1}}\right)^{2 / 3}\left[(1-\xi)-\frac{n_{j-1}^{2}}{\gamma_{j-1}^{2}}\right]
$$

with

$$
\xi=\left[1+\alpha_{1}\left(z+h_{j-1}\right)\right]
$$

and $C_{1}(\lambda), C_{2}(\lambda), Q_{1}(\lambda)$ and $Q_{2}(\lambda)$ are the constants. After obtaining the solutions of wave equations in different layers, the appropriate boundary conditions requiring the continuity of tangential electric and magnetic fields have been utilised to derive the field components over the surface of the proposed model. These boundary conditions in general for the interface lying between $j$-th and $(j+1)$-th layer can be written as (Ward, 1967): 


$$
\begin{aligned}
& \gamma_{j}^{2} \cdot \pi_{x j}=\gamma_{j+1}^{2} \cdot \pi_{x j+1}, \\
& \frac{\partial \pi_{x j}}{\partial z}=\frac{\partial \pi_{x j+1}}{\partial z}, \\
& \pi_{z j}=\pi_{z j+1}, \\
& \left(\frac{\partial \pi_{x j}}{\partial x}+\frac{\partial \pi_{z j}}{\partial z}\right)=\left(\frac{\partial \pi_{x j+1}}{\partial x}+\frac{\partial \pi_{z j+1}}{\partial z}\right) .
\end{aligned}
$$

The constants $A(\lambda), B_{j 1}(\lambda), B_{j 2}(\lambda)$ for all $j=1,2,3, \ldots, j-1, j+1, \ldots, N-1 ; C_{1}(\lambda), C_{2}(\lambda)$ and $D(\lambda)$ are evaluated using the boundary conditions given by Eqs. (23) and (24), at different interfaces. Application of these boundary conditions at $N$ interfaces gives rise to a system of $2 N$ linear equations, with the help of which one has to find out the value of $2 N$ unknowns. Similarly, the constants $P_{0}(\lambda), P_{j 1}(\lambda), P_{j 2}(\lambda)$, for all $j=1,2,3, \ldots, j-1, j+1, \ldots, N-1 ; Q_{1}(\lambda), Q_{2}(\lambda)$ and $P_{N}(\lambda)$ are evaluated using the boundary conditions given by Eqs. (23) through (26). The application of these boundary conditions would also give rise to another system of $2 N$ linear equations to find out the values of these constants. Thereafter, the field components over the surface of the model with desired number of layers and presumed conductivity variation can be obtained.

In the following section, the solution for the three layer model with intermediate inhomogeneous layer having linearly varying conductivity, acting as transition zone between the top and bottom homogeneous layers is presented.

Solving the systems of linear equations for such three layer earth model to obtain the values of required constants and substituting the values of these constants in respective equations, we get the expressions of the $\pi_{x}$ and $\pi_{z}$ components in the air and in the top layer as

$$
\begin{gathered}
\pi_{x 0}=M \int_{0}^{\infty} \frac{\lambda}{n_{0}}\left[e^{-n_{0}|z-h|}+\left\{\frac{\left(n_{0} \gamma_{1}^{2}-n_{1} \gamma_{0}^{2}\right)+\left(n_{0} \gamma_{1}^{2}+n_{1} \gamma_{0}^{2}\right) \cdot W e^{-2 n_{1} h_{1}}}{\left.\left.\left(n_{0} \gamma_{1}^{2}+n_{1} \gamma_{0}^{2}\right)+\left(n_{0} \gamma_{1}^{2}-n_{1} \gamma_{0}^{2}\right) \cdot W e^{-2 n_{1} h_{1}}\right\} \cdot e^{-n_{0}(z+h)}\right] \cdot J_{0}(\lambda \rho) d \lambda,}\right.\right. \\
\pi_{z 0}=-2 M \cos \phi \int_{0}^{\infty} \frac{\left(\gamma_{1}^{2}-\gamma_{0}^{2}\right)\left(1+W e^{-2 n_{1} h_{1}}\right)^{2} e^{-n_{0}(z+h)}}{\left[\left(n_{0}+n_{1}\right)+\left(n_{0}-n_{1}\right) W e^{-2 n_{1} h_{1}}\right]\left[\left(n_{0} \gamma_{1}^{2}+n_{1} \gamma_{0}^{2}\right)+\left(n_{0} \gamma_{1}^{2}-n_{1} \gamma_{0}^{2}\right) W e^{-2 n_{1} h_{1}}\right]} \\
\pi_{x 1}=2 M \int_{0}^{\infty} \frac{\lambda e^{-n_{0} h} \cdot \gamma_{0}^{2}\left[e^{n_{1} z}+W e^{-2 n_{1} h_{1}} e^{-n_{1} z}\right]}{\left[\left(n_{0} \gamma_{1}^{2}+n_{1} \gamma_{0}^{2}\right)+\left(n_{0} \gamma_{1}^{2}-n_{1} \gamma_{0}^{2}\right) W e^{-2 n_{1} h_{1}} \cdot J_{0}(\lambda \rho) d \lambda\right.}, \\
\pi_{z 1}=-2 M \cos \phi \int_{0}^{\infty}\left[\frac{\left(\gamma_{1}^{2}-\gamma_{0}^{2}\right)\left[e^{n_{1} z}+W e^{-2 n_{1} h_{1}} e^{-n_{1} z}\right]\left[1+W e^{-2 n_{1} h_{1}}\right]}{\left[\left(n_{0}+n_{1}\right)+\left(n_{0}-n_{1}\right) W e^{-2 n_{1} h_{1}}\right]\left[\left(n_{0} \gamma_{1}^{2}+n_{1} \gamma_{0}^{2}\right)+\left(n_{0} \gamma_{1}^{2}-n_{1} \gamma_{0}^{2}\right) W e^{-2 n_{1} h_{1}}\right]}\right] \\
\cdot \lambda^{2} J_{1}(\lambda \rho) d \lambda
\end{gathered}
$$


where

$$
\begin{aligned}
& W=[(i+v) /(i-v)], \\
& v=\left[\frac{J_{2 / 3}\left(\frac{2}{3} \xi_{1}\right)-u \cdot J_{-2 / 3}\left(\frac{2}{3} \xi_{1}\right)}{J_{-1 / 3}\left(\frac{2}{3} \xi_{1}\right)+u \cdot J_{1 / 3}\left(\frac{2}{3} \xi_{1}\right)}\right], \\
& u=\left[\frac{i J_{2 / 3}\left(\frac{2}{3} \xi_{2}\right)-J_{-1 / 3}\left(\frac{2}{3} \xi_{2}\right)}{i J_{-2 / 3}\left(\frac{2}{3} \xi_{2}\right)+J_{1 / 3}\left(\frac{2}{3} \xi_{2}\right)}\right], \\
& \xi_{1}=\left(\frac{i^{3} n_{1}^{3}}{\left(\alpha_{1} \gamma_{1}^{2}\right)}\right) ; \quad \xi_{2}=\left(\frac{i^{3} n_{3}^{3}}{\left(\alpha_{1} \gamma_{1}^{2}\right)}\right)
\end{aligned}
$$

and

$$
\gamma_{2}^{2}(z)=\gamma_{1}^{2}\left[1+\alpha_{1}\left(z+h_{1}\right)\right]
$$

The expressions (27) and (28) for $h=0$; and $h_{1} \rightarrow \infty$; i.e. Hertz vector components $\pi_{x}$ and $\pi_{z}$ for homogeneous earth, when the source is on the surface of the earth, reduce to

$$
\pi_{x 0_{(h=0)}}=2 M \int_{0}^{\infty} \frac{\gamma_{1}^{2}}{\left(n_{0} \gamma_{1}^{2}+n_{1} \gamma_{0}^{2}\right)} e^{-n_{0} z} \cdot \lambda J_{0}(\lambda \rho) d \lambda
$$

and

$$
\pi_{z 0_{(h=0)}}=-2 M \cos \phi \int_{0}^{\infty} \frac{\left(\gamma_{1}^{2}-\gamma_{0}^{2}\right)}{\left(n_{0}+n_{1}\right)\left(n_{0} \gamma_{1}^{2}+n_{1} \gamma_{0}^{2}\right)} e^{-n_{0} z} \cdot \lambda^{2} J_{1}(\lambda \rho) d \lambda
$$

These expressions (31) and (32) are in agreement with those found by Wait (1953). Further, taking $\gamma_{0}^{2}=$ 0 , and $h_{1} \rightarrow \infty$; in Eqs. (29) and (30), the Hertz vector components $\pi_{x}$ and $\pi_{z}$ for homogeneous earth, in top layer reduce to

$$
\pi_{x 1}=0
$$

and

$$
\pi_{z 1}=-2 M \cos \phi \int_{0}^{\infty} \frac{\lambda}{\lambda+n_{1}} e^{n_{1} z} \cdot J_{1}(\lambda \rho) d \lambda
$$


These expressions (33) and (34) are identical to those given by Ward (1967). Thus, the results (31), (32), (33) and (34) provide the confirmation for the results obtained in Eqs. (27)-(30).

The electric and magnetic field components in the air can be derived from Eq. (9). In air, on the earth surface, the field components are:

$$
\begin{aligned}
& E_{x 0}=-i \omega \mu_{0} \cdot M \cdot \sin 2 \phi\left[T_{1}-\frac{T_{4}}{\rho}\right], \\
& E_{y 0}=2 i \omega \mu_{0} \cdot M\left[\cos ^{2} \phi\left(T_{1}-\frac{T_{4}}{\rho}\right)\right], \\
& E_{z 0}=2 i \omega \mu_{0} \cdot M \cdot \sin \phi \cdot T_{3}, \\
& H_{x 0}=-2 M \cdot \cos { }^{2} \phi\left[T_{5}-\frac{T_{6}}{\rho}\right], \\
& H_{y 0}=-M \cdot \sin 2 \phi\left[T_{5}-\frac{T_{6}}{\rho}\right], \\
& H_{z 0}=-2 M \cdot \cos \phi \cdot T_{2}
\end{aligned}
$$

where

$$
\begin{aligned}
& T_{1}=\int_{0}^{\infty} \frac{\lambda^{2}}{\left(\lambda+n_{1}\right)}\left[\frac{1+W e^{-2 n_{1} h_{1}}}{1+\left(\frac{\lambda-n_{1}}{\lambda+n_{1}}\right) W e^{-2 n_{1} h_{1}}}\right] \cdot J_{0}(\lambda \rho) d \lambda, \\
& T_{2}=\int_{0}^{\infty} \frac{\lambda^{3}}{\left(\lambda+n_{1}\right)}\left[\frac{1+W e^{-2 n_{1} h_{1}}}{1+\left(\frac{\lambda-n_{1}}{\lambda+n_{1}}\right) W e^{-2 n_{1} h_{1}}}\right] \cdot J_{1}(\lambda \rho) d \lambda, \\
& T_{3}=\int_{0}^{\infty} \lambda \cdot J_{1}(\lambda \rho) d \lambda, \\
& T_{4}=\int_{0}^{\infty} \frac{\lambda}{\left(\lambda+n_{1}\right)}\left[\frac{1+W e^{-2 n_{1} h_{1}}}{1+\left(\frac{\lambda-n_{1}}{\lambda+n_{1}}\right) W e^{-2 n_{1} h_{1}}}\right] \cdot J_{1}(\lambda \rho) d \lambda,
\end{aligned}
$$




$$
\begin{aligned}
& T_{5}=\int_{0}^{\infty} \frac{\lambda^{2} n_{1}}{\left(\lambda+n_{1}\right)}\left[\frac{1-W e^{-2 n_{1} h_{1}}}{1+\left(\frac{\lambda-n_{1}}{\lambda+n_{1}}\right) W e^{-2 n_{1} h_{1}}}\right] \cdot J_{0}(\lambda \rho) d \lambda, \\
& T_{6}=\int_{0}^{\infty} \frac{\lambda n_{1}}{\left(\lambda+n_{1}\right)}\left[\frac{1-W e^{-2 n_{1} h_{1}}}{1+\left(\frac{\lambda-n_{1}}{\lambda+n_{1}}\right) W e^{-2 n_{1} h_{1}}}\right] \cdot J_{1}(\lambda \rho) d \lambda .
\end{aligned}
$$

\section{Computational Scheme}

The computation of infinite integrals $T_{1}, T_{2}, T_{3}, T_{4}, T_{5}$ and $T_{6}$ occurring in the expressions of various em field components derived in last section is quite intricate and complex. This problem has, however been overcome by expressing these infinite integrals involving Bessel function of order 0 and 1 , in terms of Hankel transforms of order 0 and 1 . Accordingly, the Hankel transform of the Kernel $K(\lambda)$ of integer order $n$ is defined as (Anderson, 1979)

$$
k(b)=\int_{0}^{\infty} k(\lambda) \cdot J_{n}(b \lambda) d \lambda ; b>0
$$

where $J_{n}$ is the Bessel function of the first kind of order $n$. The transform argument $b>0$ is real but $k(\lambda)$ and therefore $k(b)$ may be complex function of real variable.

Anderson (1979) presented a linear digital filter algorithm for rapid and accurate numerical evaluation of the Hankel transforms of order 0 and 1, for the related kernel functions and illustrated its utility with some examples. The kernel functions used in the above algorithm called ZHANKS, must be a continuous bounded complex function as the argument increases. Therefore, in order to use the algorithm, ZHANKS for the present problem, the convergence of the kernel functions is tested. In case of slow convergence or divergence nature, they are made to converge rapidly by subtracting or adding a known integral expression under the integral sign and subsequently adjusting its equivalent analytic expression outside the integral sign. As an illustration, the expression of $T_{1}$ is adjusted as

$$
T_{1}=\left[\int_{0}^{\infty}\left\{\frac{1}{1+\left(R_{N} / R_{M}\right) \cdot\left(n_{1} / \lambda\right)}-0.5\right\} \lambda J_{0}(\lambda \rho) d \lambda+0.5 \int_{0}^{\infty} \lambda J_{0}(\lambda \rho) d \lambda\right]
$$

with

$$
R_{M}=1+W e^{-2 n_{1} h_{1}} ; \quad R_{N}=1-W e^{-2 n_{1} h_{1}}
$$

and under limiting condition,

$$
\frac{1}{1+\left(R_{N} / R_{M}\right) \cdot\left(n_{1} / \lambda\right)} \rightarrow \frac{1}{2} ; \quad \text { as } \lambda \rightarrow \infty
$$


In this Eq. (48), the first part is evaluated using the algorithm ZHANKS (Anderson, 1979), whereas the second part is directly evaluated using the relation (Watson, 1944)

$$
\int_{0}^{\infty} \lambda J_{0}(\lambda \rho) d \lambda=0
$$

In similar manner, other integral expressions are evaluated using the program, ZHANKS and appropriate direct relations. Further, for evaluating the Bessel functions involved in the kernel functions standard expressions for them have been used (McLachlan, 1955).

\section{Results and Discussions}

In order to investigate the effect of linear variation of subsurface conductivity on em response, different three layer models have been chosen with the presumption that the conductivity of the top layer gradually merges with that of the basement or substratum following the linear variation in the intermediate transition layer. Abramovici and Chlamtac (1978) have presented the computational results for transition layer models with the linear variation of conductivity for vertical magnetic dipole source and have pointed out that the dependence between the em field component curves and the model is not very clear, as the general trend of the curves looks similar. However, much stronger correlation between the curves and the models is noticeable by representing not the fields themselves but the ratios of field components of the model to corresponding components for a homogeneous half space, possessing the conductivity of the top layer. Further, they have also observed that such absolute amplitude ratio and the phase curves show a similar behaviour for all the components, except for the beginning portion of the curves.

In view of the above observations, it is pertinent here to perform only the computations for the ratio of the absolute values of the amplitude of different field components and the corresponding components for homogeneous half space with top layer conductivity; we hereafter call them absolute-amplitude ratio values. We will investigate the effect of transition layer thickness and the conductivity contrast between the top and bottom layers. These ratios are computed as a function of numerical distance, $d_{1}=\sqrt{\omega \mu_{0} \sigma_{1}} \cdot \rho$, which was introduced by Abramovici and Chlamtac (1978). Here, $\rho$ is the separation between the transmitter and receiver and all the other symbols are defined earlier.

The variation of the absolute amplitude ratio of the $H_{x}, E_{y}$ and $H_{z}$ components with $d_{1}$, for different transition layer thicknesses $h=\left(h_{2}-h_{1}\right) / h_{1}=0.5,1.0,3.0,5.0$ and 10.0 are presented in Figs. 2(a), 3(a) and 4(a), and for different conductivity contrast between the top and bottom layers are shown in Figs. 2(b), 3(b) and 4(b), for the models inset in the figures. A general observation of these curves reveals that for very small and very large values of numerical distance, the curves approach to a value very close to unity whereas for the intermediate values of numerical distance, the curves show their characteristic variations for different components showing the effects of subsurface structures and the model characteristics.

From Fig. 2(a), showing the variation of the absolute-amplitude ratio of $H_{x}$ component with $d_{1}$ for the relative thicknesses of the transition layer, it is observed that the curves have values close to unity for small values of $d_{1}(<0.6)$. At $d_{1}=0.6$, they start to depart from the unity value and form a maximum peak at $\left(4<d_{1}<8\right)$. Thereafter, the curves decrease smoothly and converge again to the values close to unity for $d_{1}>50$. The curves are sharp and have well defined peaks. As the thickness of transition layer increases, the maximum peak of the curve decreases in amplitude and width. The effect of variation of conductivity contrast on the absolute-amplitude ratio curve of $H_{x}$ component shown in Fig. 2(b) reflects similar trend. Further, it is noticed that with the decrease in the conductivity contrast between the top and bottom layers the maximum peak of the curve increases in amplitude and width.

Figure 3(a), showing the variation of $\left|E_{y n}\right| /\left|E_{y n}{ }^{0}\right|$ with $d_{1}$ for the various thicknesses of the transition layer, reflects that for very small values of $d_{1}(<1.0)$, the curves have values close to unity. Then, they start to decrease and form a minimum peak around $\left(8<d_{1}<20\right)$. Thereafter, they increase smoothly and attain the values close to unity again at $\left(d_{1}>10^{2}\right)$. Further, with the increase in the thickness of transition layer, 
(a)

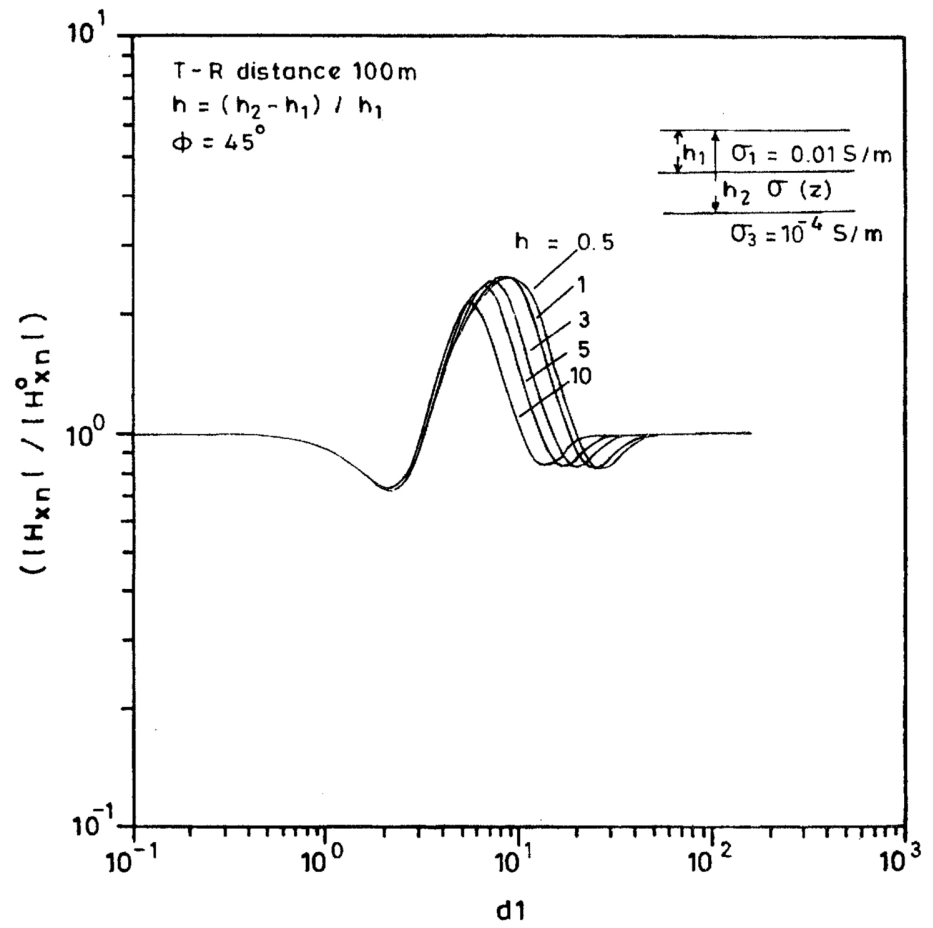

(b)

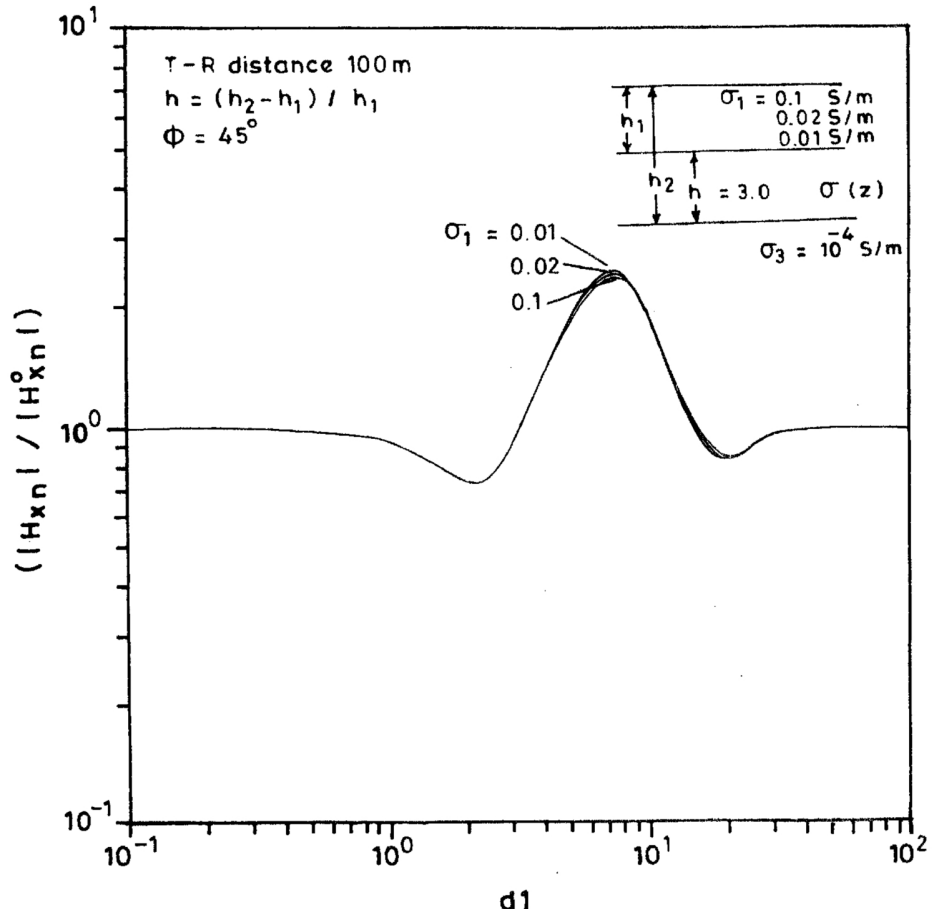

Fig. 2. Plot of the $\left|H_{x n}\right| /\left|H_{x n}{ }^{0}\right|$ vs. $d_{1}$, showing the effect of variation of (a) transition layer thickness, and (b) conductivity contrast between the top and bottom layers. 
(a)

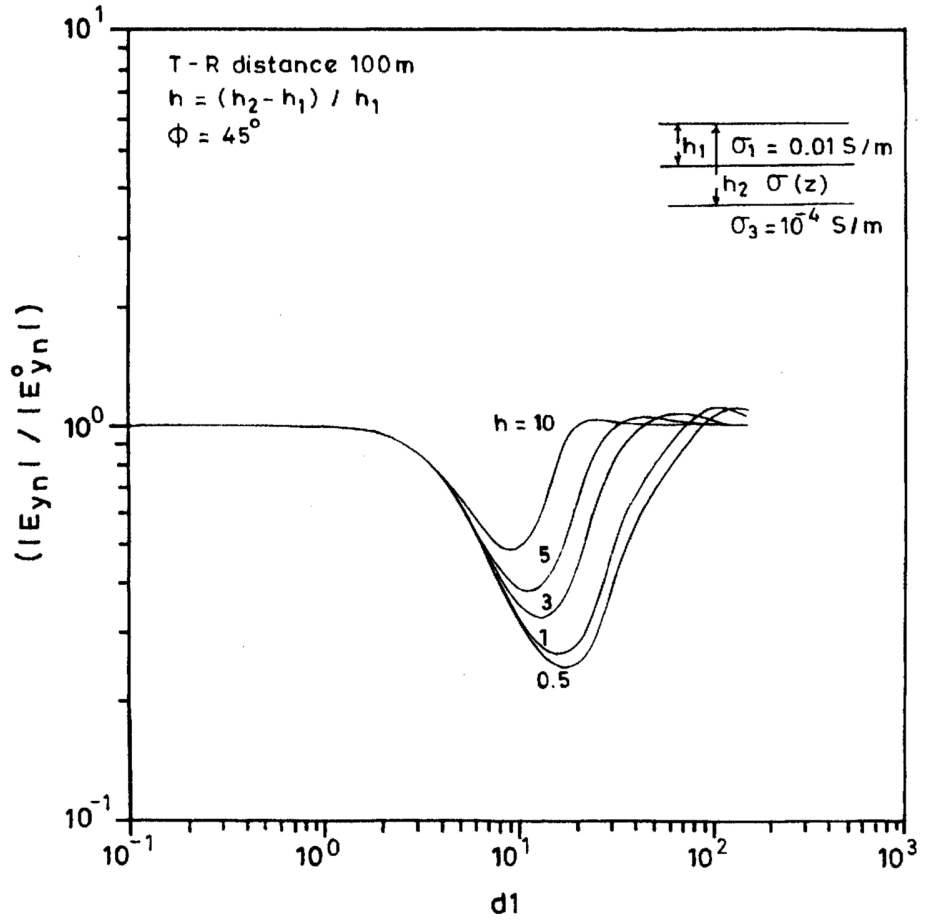

(b)

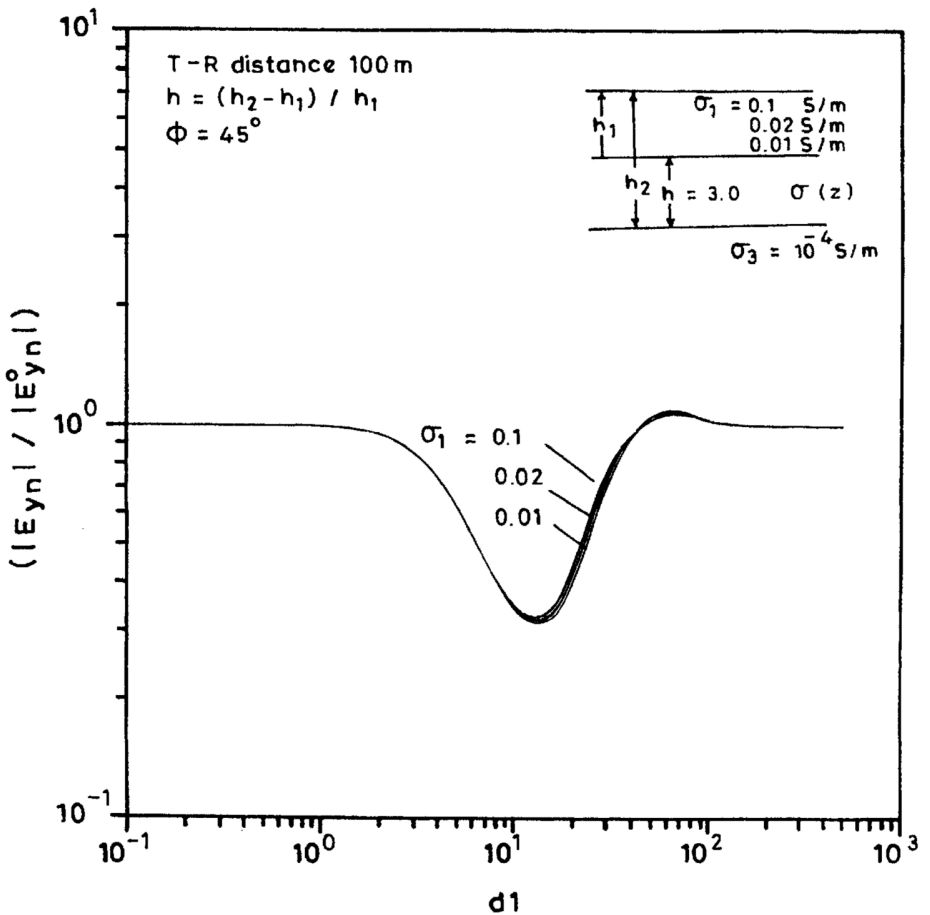

Fig. 3. Plot of the $\left|E_{y n}\right|\left|E_{y n}{ }^{0}\right|$ vs. $d_{1}$, showing the effect of variation of (a) transition layer thickness, and (b) conductivity contrast between the top and bottom layers. 
(a)

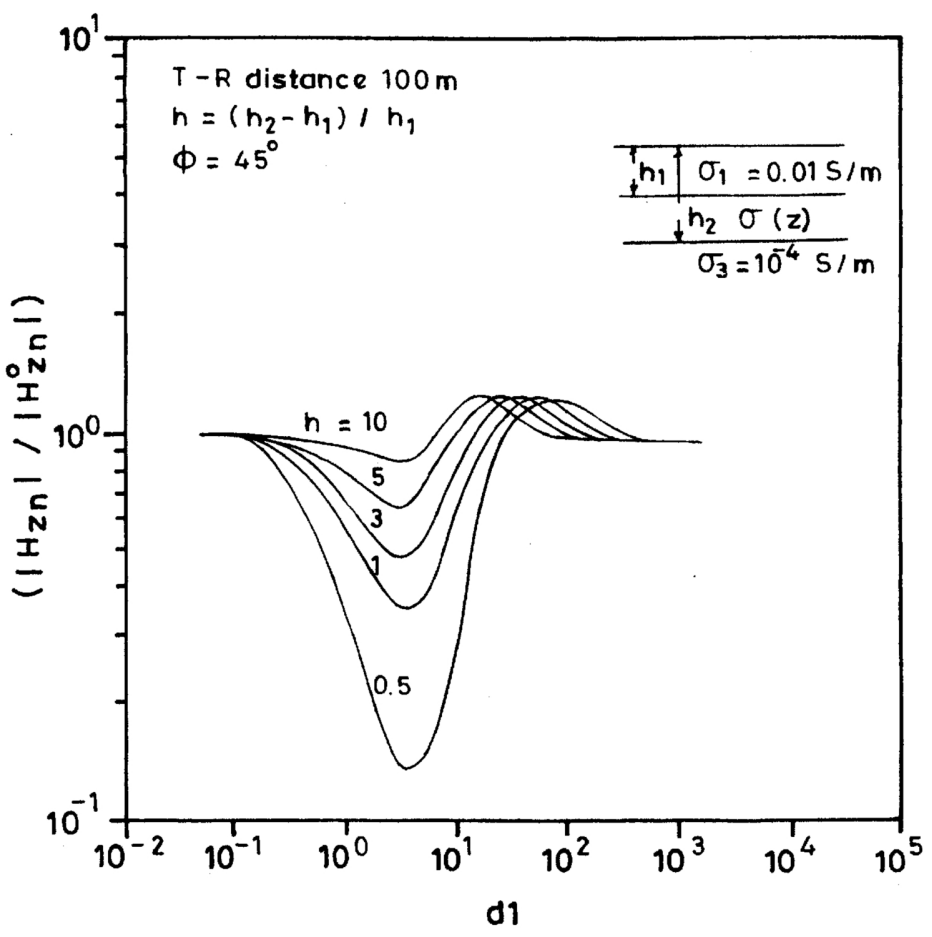

(b)

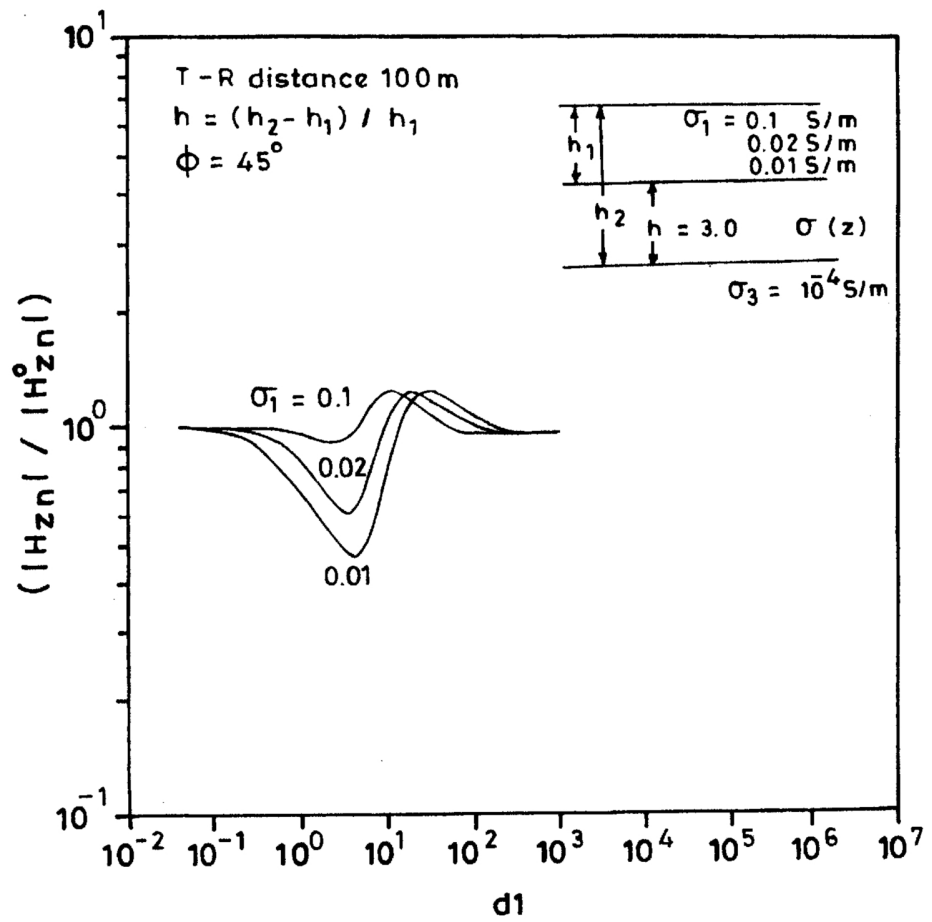

Fig. 4. Plot of the $\left|H_{z n}\right| /\left|H_{z n}{ }^{0}\right|$ vs. $d_{1}$, showing the effect of variation of (a) transition layer thickness, and (b) conductivity contrast between the top and bottom layers. 
the amplitude and width of the minimum peak decrease. From Fig. 3(b), showing the effect of variation of conductivity contrast on the absolute-amplitude ratio curve of $E_{y}$ component, it is observed that with the decrease in the conductivity contrast between the top and bottom layers, the amplitude and width of the minimum peak increase.

From Fig. 4(a), showing the variation of the absolute-amplitude ratio of $H_{z}$ component with $d_{1}$ for the various thicknesses of the transition layer, it is clear that the curves have values close to unity for small values of $d_{1}(<0.1)$ and then at $d_{1}=0.1$, they start decreasing and form a minimum peak at $d_{1}=0.6$. After this, the curves start to increase and attain the values close to unity again for $d_{1}\left(>5 \times 10^{2}\right)$. The curves make a valley-like shape. Further, it is noticed that with the increase in the thickness of transition layer, there is a decrease in the amplitude and width of the minimum peak. Figure 4(b), showing the effect of variation of conductivity contrast on the absolute-amplitude ratio curve of $H_{z}$ component, depicts that as the conductivity contrast between the top and bottom layers decreases, the peak of the minimum increases in amplitude and width.

\section{Conclusions}

The electromagnetic field response of a horizontal magnetic dipole placed on the surface of a vertically inhomogeneous earth model having linear variation of conductivity with depth in the inhomogeneous layer have been analytically and numerically evaluated. The analytical solutions involve integral equations and the program used for numerical computations is based on the linear digital filter theory, in contrast to those involving differential equations and Runge-kutta method of numerical computation.

From the plots of the absolute-amplitude ratio of $H_{x}, E_{y}$ and $H_{z}$ against the numerical distance showing the effects of variation of relative transition layer thickness and conductivity contrast between the top and bottom layers, it is observed that all the curves have values close to unity for very small and large values of numerical distance and reflect their characteristic variations mainly in the intermediate range of numerical distance values. The peaks of the curves decrease in amplitude and width with the increase in the thickness of the transition layer. Moreover, it is also observed that with the decrease in the conductivity contrast between the top and bottom layers, the peak of the curves increases in amplitude and width.

The authors thank reviewers for their constructive comments which greatly improved the quality of the manuscript. One of us (NPS) is thankful to the Council of Scientific and Industrial Research, New Delhi, India, for the financial support in the form of research fellowship.

\section{REFERENCES}

Abramovici, F. and M. Chlamtac, Fields of a vertical magnetic dipole over a vertically inhomogeneous earth, Geophysics, 43, 5, 954-966, 1978.

Anderson, W. L., Numerical integration of related Hankel transforms of order 0 and 1 by adaptive digital filtering, Geophysics, 44, 7, 1287-1305, 1979.

Chlamtac, M. and F. Abramovici, The electromagnetic fields of a horizontal dipole over vertically inhomogeneous and anisotropic earth, Geophysics, 46, 6, 904-915, 1981.

Keller, G. V., Electrical characteristics of the earth's crust, in Electromagnetic Probing in Geophysics, edited by J. R. Wait, 13 pp., Golden Press, 1971.

Mallick, K. and A. Roy, Resistivity sounding on a two layer earth with transitional boundary, Geophys. Prospect., 16, 436-446, 1968.

Mallick, K. and A. Roy, Vertical magnetic dipole over transitional earth, Geophys. Prospect., 19, 388-394, 1971.

McLachlan, N. W., Bessels Function for Engineers, Oxford Univ. Press, New York, N.Y., 1955.

Sommerfeld, A., Partielle Differentialgleichungen der Physik, Akademische verlagsgesellschaft I, Auflage, Leipzig, 1947.

Wait, J. R., Propagation of radio waves over a stratified ground, Geophysics, 18, 2, 416-422, 1953.

Ward, S. H., Electromagnetic theory for geophysical applications, in Mining Geophysics Volume-II: Theory, SEG, 1967.

Watson, G. N., Theory of Bessel Functions, 386 pp., Cambridge Univ. Press, London, 1944. 